Research Paper

\title{
Cardiomyogenic Differentiation of Human Dental Follicle-derived Stem Cells by Suberoylanilide Hydroxamic Acid and Their In Vivo Homing Property
}

\author{
Iel-Yong Sung ${ }^{1 *}$, Han-Na Son ${ }^{1 *}$, Imran Ullah², Dinesh Bharti², Ju-Mi Park², Yeong-Cheol Cho ${ }^{1}$, June-Ho \\ Byun $^{3}$, Young-Hoon Kang ${ }^{3,4}$, Su-Jin Sung, ${ }^{4}$ Jong-Woo Kim5, Gyu-Jin Rho ${ }^{2 凶}$, and Bong-Wook Park ${ }^{3,4} \bowtie$ \\ 1. Department of Oral and Maxillofacial Surgery, College of Medicine, Ulsan University, Ulsan, Republic of Korea \\ 2. Department of Theriogenology and Biotechnology, College of Veterinary Medicine and Research Institute of Life Science, Gyeongsang National University, \\ Jinju, Republic of Korea \\ 3. Department of Dentistry, Gyeongsang National University School of Medicine and Institute of Health Science, Jinju, Republic of Korea \\ 4. Department of Oral and Maxillofacial Surgery, Changwon Gyeongsang National University Hospital, Changwon, Republic of Korea \\ 5. Department of Thoracic and Cardiovascular Surgery, Gyeongsang National University School of Medicine and Changwon Gyeongsang National University \\ Hospital, Changwon, Republic of Korea \\ * These authors contributed equally to this work \\ $\llbracket$ Corresponding authors: Bong-Wook Park; parkbw@gnu.ac.kr or Gyu-Jin Rho; jinrho@gnu.ac.kr
}

(1) Ivyspring International Publisher. Reproduction is permitted for personal, noncommercial use, provided that the article is in whole, unmodified, and properly cited. See http://ivyspring.com/terms for terms and conditions.

Received: 2016.06.23; Accepted: 2016.09.01; Published: 2016.10.18

\begin{abstract}
The purpose of the present study was to investigate the in vitro cardiomyogenic differentiation potential of human dental follicle-derived stem cells (DFCs) under the influence of suberoylanilide hydroxamic acid (SAHA), a member of the histone deacetylase inhibitor family, and analyze the in vivo homing capacity of induced cardiomyocytes (iCMs) when transplanted systemically. DFCs from extracted wisdom teeth showed mesenchymal stem cell (MSC) characteristics such as plate adherent growing, expression of MSC markers (CD44, CD90, and CD105), and mesenchymal lineage-specific differentiation potential. Adding SAHA to the culture medium induced the successful in vitro differentiation of DFCs into cardiomyocytes. These iCMs expressed cardiomyogenic markers, including alpha-smooth muscle actin ( $\alpha$-SMA), cardiac muscle troponin T (TNNT2), Desmin, and cardiac muscle alpha actin (ACTCl), at both the mRNA and protein level. For the assessment of homing capacity, PKH26 labeled iCMs were intraperitoneally injected $\left(1 \times 10^{6}\right.$ cells in $100 \mu \mathrm{L}$ of PBS) into the experimental mice, and the ratios of $\mathrm{PKH} 26$ positive cells to the total number of injected cells, in multiple organs were determined. The calculated homing ratios, 14 days after systemic cell transplantation, were $5.6 \pm 1.0 \%, 3.6 \pm 1.1 \%$, and $11.6 \pm 2.7 \%$ in heart, liver, and kidney respectively. There was no difference in the serum levels of interleukin-2 and interleukin-10 at 14 days after transplantation, between the experimental (iCM injected) and control (no injection or PBS injection) groups. These results demonstrate that DFCs can be an excellent source for cardiomyocyte differentiation and regeneration. Moreover, the iCMs can be delivered into heart muscle via systemic administration without eliciting inflammatory or immune response. This can serve as the pilot study for further investigations into the in vitro cardiomyogenic differentiation potential of DFCs under the influence of SAHA and the in vivo homing capacity of the iCMs into the heart muscle, when injected systemically.
\end{abstract}

Key words: human dental follicle-derived stem cells, suberoylanilide hydroxamic acid

\section{Introduction}

Cardiovascular disease has been one of the major global health problems. Specifically, ischemic myocardial injury is the main cause of heart failures
$[1,2]$. Many therapeutic methods are used to treat cardiovascular disorders. However, the conventional management of heart failure has very little effect on 
the recovery of the injured heart cells. One of the potential new strategies for ischemic heart diseases is cell based cardiac muscle regeneration by transplanting cardiomyocytes differentiated in vitro from stem cells [3]. Mesenchymal stem cells (MSCs) have been shown to have excellent potential to differentiate into cardiomyocytes in vitro [3, 4]. MSCs have the property of self-renewal, are easily available, constrained by few ethical issues, and can be cultivated in vitro for a long time. MSCs have been isolated from various adult tissues, such as bone marrow, fat, skin, blood, umbilical cord, and dental tissues [5, 6]. Among them, bone marrow-derived MSCs were most frequently used and studied for the in vitro cardiomyogenic differentiation potential and the in vivo therapeutic efficiency after cell transplantation [4, 7]. However, MSCs from dental tissues have been the focus of modern stem cell research because of the ease of harvesting, self-renewal, and multilineage differentiation potential [8-10]. Especially, dental follicle, pulp, and root apical papilla of the extracted wisdom teeth showed the highest potential as MSC sources for various tissue regenerations [8]. MSCs from dental pulp tissue of deciduous teeth could be differentiated in vitro into cardiomyocytes and expressed cardiomyocyte specific markers at a high level during the course of differentiation [11].

Several methods have been used in the research area of cardiomyogenic differentiation of stem cells; induction with biochemical substances, cell culture in simulated myocardial microenvironment, and genetic modification [4]. Among them, using various biochemical reagents to induce the differentiation of stem cells into cardiomyocytes has proven to be a simple and effective method. Several chemical and biochemical agents such as 5-azacytidine (5-aza), bone morphogenetic protein-2 (BMP-2), angiotensin-II, and dimethyl sulfoxide (DMSO) have been used for inducing cardiomyogenic differentiation in vitro [4, 14]. Of these, 5-aza, a DNA methylation inhibitor, has been the most widely investigated chemical agent. Many researchers showed myotube-like structure, spontaneous beating, and cardiac-specific gene expressions in the cardiomyogenic cells derived from 5-aza treated MSCs [12-14]. However, low differentiation efficiency, cellular toxicity, and cell death due to fat deposition in cytoplasm have been reported as the drawbacks of 5-aza induction protocol $[4,15,16]$.

There have been several attempts to differentiate cardiomyocytes from stem cells with various chemicals other than 5-aza $[4,7,14,16,17]$. One of them, suberoylanilide hydroxamic acid (SAHA), a member of histone deacetylase (HDAC) inhibitor family, showed potential for effectively inducing the cardiomyogenic differentiation of bone marrow derived MSCs in vitro [16]. Moreover, a recent study reported that inhibition of HDAC improves myocardial function, protects the heart against myocardial injury, and stimulates angiomyogenesis in the heart muscle [18].

One of the critical aspects of cell therapy strategies for cardiomyogenic regeneration is the cell delivery method. Since heart is a beating organ, open-heart surgery is needed for direct and localized introduction of therapeutic cells into the injured heart tissue. To simplify cell therapy that yields effective therapeutic results in heart diseases, systemic cell delivery system should be considered, including intravenous (IV) or intraperitoneal (IP) injection of the cells. Many studies have reported that MSCs had homing capacity and localize to the injured organs or tissues, following systemic cell delivery methods such as IV or IP injection [19-22]. Homing of MSCs is the result of interaction with host tissues accompanied by the secretion of trophic factors [22]. However, the exact mechanism by which MSCs migrate and home to the injured site is still unknown, although it is believed that specific chemokines and their receptors are involved in the process [20]. Notably, the in vivo homing property of the cells differentiated from stem cells has not been well studied.

In the present study, we isolated MSCs from human dental follicles (DFCs) from the extracted wisdom teeth, and differentiated them into cardiomyocytes in vitro using SAHA induction media. The characteristics of induced cardiomyocytes (iCMs) from DFCs were analyzed with respect to the expression of cardiomyogenic markers at gene and protein levels. The iCMs were intraperitoneally injected into the experimental mice and the in vivo cell homing to heart, liver, and kidney was quantitated at 14 days after cell injection. Immune response to systemic cell injection was analyzed by the changes in serum IL-2 and IL-10 levels.

\section{Materials and Methods}

\section{Chemicals, media, and approval of animal experiments}

Unless otherwise specified, all chemicals were purchased from Sigma-Aldrich (St. Louis, MO, USA), and all media were from Gibco (Invitrogen, Grand Island, NY, USA). For all media, the $\mathrm{pH}$ was adjusted to 7.4 and the osmolality was adjusted to 280 $\mathrm{mOsm} / \mathrm{kg}$. Animal experiments using mice were approved by the Animal Center for Medical Experimentation at Gyeongsang National University. 


\section{Isolation and culture of human dental MSCs}

Human dental follicle-derived MSCs (DFCs) were isolated from the dental follicles of extracted wisdom teeth and cultured as per previously described protocols [8-10]. Briefly, after obtaining informed consents, the wisdom teeth from 15 patients (8 men and 7 women; aging between 18-22 years), who were undergoing wisdom teeth extraction at the Department of Oral and Maxillofacial Surgery at Gyeongsang National University Hospital, were collected in accordance with the approved guidelines set by GNUHIRB-2012-09-004-002. The extracted wisdom teeth samples were aseptically transferred to the laboratory and rinsed several times with $1 \%$ Pen-Strep (Penicillin-Streptomycin) containing DPBS. Dental follicles were carefully separated from the tooth surface by using a sterile scalpel. Dental follicle tissues were minced into small pieces and digested with $1 \mathrm{mg} / \mathrm{ml}$ collagenase type I (Millipore, CA, USA) in DPBS at $37^{\circ} \mathrm{C}$ for $30 \mathrm{~min}$ with frequent gentle agitation. After complete digestion, single cell suspensions were prepared by successive filtrations using $100-\mu \mathrm{m}$ and $40-\mu \mathrm{m}$ nylon cell strainers. Filtered cell suspensions were centrifuged at $500 \times g$ for $5 \mathrm{~min}$, the cell pellet was re-suspended in Advanced Dulbecco's Modified Eagle Medium (ADMEM) supplemented with $10 \%$ fetal bovine serum (FBS) and seeded into 25 T-flasks (Nunc ${ }^{\mathrm{TM}}$, Roskilde, Denmark). Cultures were incubated at $37^{\circ} \mathrm{C}$ in a humidified atmosphere of $5 \% \mathrm{CO}_{2}$ in air. Media was changed every 3 days until the primary cultures reached $80-90 \%$ confluence. Confluent cells were then harvested with $0.25 \%(\mathrm{w} / \mathrm{v})$ trypsin EDTA solution and sub-cultured until passage 3 for further experimentation.

\section{Characterization of DFCs}

\section{Expression of early transcription markers}

DFCs at passage 3 were harvested with $0.25 \%$ trypsin EDTA treatment for $5 \mathrm{~min}$ at $37^{\circ} \mathrm{C}$. Harvested cells were resuspended in 10\% FBS containing ADMEM for trypsin inactivation followed by centrifugation at $500 \times g$ for $5 \mathrm{~min}$. The cell pellet was recovered and used for total RNA extraction. The mRNA levels of pluripotency and early transcription markers, Oct4, Sox2, and Nanog, were assessed by quantitative real-time PCR (RT-qPCR) and confirmed by gel electrophoresis.

\section{Analysis of Population Doubling Time}

A total of $2 \times 10^{3}$ cells were seeded in each well of 24 well plates (Nunc ${ }^{\mathrm{TM}}$ ) to determine population doubling time (PDT) of DFCs. Cells were grown for a total of 14 days, taking cell counts at two day intervals. For cell counts, cells were harvested using
$0.25 \%$ trypsin EDTA. PDT of DFCs was calculated by using the formula, PDT $=\mathrm{t}(\log 2) /(\log N \mathrm{t}-\log \mathrm{N} 0)$, where $\mathrm{N} 0$ and $\mathrm{Nt}$ are the number of cells seeded and the number of cells at time $t$ respectively where $t$ denotes culture duration. Experiments were performed in triplicate to reduce the error rate.

\section{Expression of cell surface markers}

MSC markers in cultured DFCs were analyzed by fluorescent activated cell sorting (FACS) method. (BD FACSCalibur, Becton Dickinson, Franklin Lakes, NJ, USA). DFCs at $80-90 \%$ confluence at passage 3, were fixed with $3.7 \%$ formaldehyde for $1 \mathrm{~h}$ followed by washing thrice with DPBS. The cells were directly labeled with fluorescein isothiocyanate (FITC)-conjugated primary antibodies [anti-mouse CD44 (1:100; BD Pharmingen ${ }^{\mathrm{TM}}$, BD Biosciences, Franklin Lakes, NJ, USA), anti-human CD 34 (1:100; BD Biosciences) anti-human CD45 (1:100; BD Biosciences)] or with unconjugated primary antibodies [mouse monoclonal IgG2a CD105 (1:100; Santa Cruz biotechnology, Inc., Dallas, TX, USA) and anti-human CD90 (1:100; BD Biosciences)]. A total of 10,000 cells were analyzed by flow cytometry using CellQuest software (Becton Dickinson).

\section{In vitro differentiations into adipocytes and osteocytes}

Adipocyte and osteocyte differentiation potential of DFCs was assessed by previously published protocols with minor modifications $[6,9]$. DFCs at passage 3 were cultured in ADMEM supplemented with 10\% FBS under adipogenic and osteogenic conditions for 21 days with media change every 3 days. Untreated cells (DFCs) were taken as control and were maintained under normal conditions in ADMEM containing 10\% FBS. Adipogenic inductive media contained $10 \mu \mathrm{M}$ dexamethasone, $10 \mu \mathrm{M}$ insulin, $500 \mu \mathrm{M}$ isobutyl methyl xanthine, and $100 \mu \mathrm{M}$ indomethacin. Adipocyte differentiation was evaluated by staining for oil droplets with Oil red O. Adipocyte differentiation was also assessed by the quantification of the mRNA levels of adipocyte-specific genes, including fatty acid binding protein 4 (FABP4), lipoprotein lipase $(L P L)$ and peroxisome proliferator-activated receptor $(P P A R \gamma)$, using RT-qPCR. Osteocyte differentiation was induced by culturing the cells in media containing $1 \mu \mathrm{M}$ dexamethasone, $10 \mathrm{mM}$ sodium $\beta$-glycerophosphate, and $0.05 \mathrm{mM}$ ascorbic acid. Osteogenic phenotype was assessed by Alizarin red and von Kossa staining for mineralized calcium deposits. The osteogenic differentiation was also evaluated by measuring the mRNA levels of osteogenic-specific genes, such as 
osteonectin $(O N)$, runt-related transcription factor-2 (RUNX-2) and bone morphogenetic protein-2 (BMP-2), by RT-qPCR.

RT-qPCR was performed according to the manufacturer's instructions. Briefly, total RNA was extracted from osteogenic and adipogenic differentiated cells and control cells (undifferentiated DFCs) by RNeasy mini kit (Qiagen, CA, USA). Total RNA concentrations were quantified by OPTIZEN 3220 UV BIO Spectrophotometer (Mecasys, Daejeon, Korea) and pure total RNAs with $2 \pm 0.2$ A260/A280 ratio were selected. Complementary DNA (cDNA) was synthesized with $1 \mu \mathrm{g}$ total RNA, mom oligo dT primers (Invitrogen, Carlsbad, USA), 10 units of RNase inhibitor (Invitrogen) and 4 units of Omniscript Reverse Transcriptase (Qiagen). DNAse I (Qiagen) treatment for $15 \mathrm{~min}$ was followed by RT-qPCR by the addition of $2 \mu \mathrm{l}$ of cDNA, $2 \mu \mathrm{l}$ of 10 $\mu \mathrm{m}$ specific primer pairs, and $16 \mu \mathrm{l}$ of distilled water in $20 \mu \mathrm{l}$ of PCR PreMix ${ }^{\mathrm{TM}}$ (iNTRO Biotechnology, Seongnam, Korea). Tyrosine 3-monooxygenase/ tryptophan 5-monooxygenase activation protein, zeta (YWHAZ) was used as the reference gene to evaluate the efficiency of reverse transcription. PCR reaction involved initial denaturation at $94^{\circ} \mathrm{C}$ for $30 \mathrm{~s}$, annealing for $30 \mathrm{~s}$, elongation at $72^{\circ} \mathrm{C}$ for $90 \mathrm{~s}$ and final primer extension at $72^{\circ} \mathrm{C}$ for $10 \mathrm{~min}$. The sequence information for primers used in the present study is shown in Table 1.

\section{In vitro cardiomyogenic differentiation of DFCs}

The cardiomyocyte differentiation of DFCs was performed using SAHA in the normal growth media. To induce cardiomyocyte differentiation, DFCs at $80 \%$ confluence at passage 3 were treated with $10 \mu \mathrm{M}$ SAHA in ADMEM media supplemented with $10 \%$ FBS. After $24 \mathrm{~h}$ of initial exposure to SAHA, the medium was replaced with fresh media containing 1 $\mu \mathrm{M}$ of SAHA. The cardiomyogenic induction was continued for 14 days with media change every 3 days. Untreated cells (DFCs) were cultured in ADMEM with 10\% FBS for the same duration to serve as control cells.

Total RNAs were isolated from the induced cardiomyocytes (iCMs) and control cells (undifferentiated DFCs) by RNeasy mini kit (Qiagen) for RT-qPCR analysis, by the method described above. Relative mRNA levels of alpha-smooth muscle actin (a-SMA), cardiac muscle troponin $T$ (TNNT2), Desmin, and cardiac muscle alpha actin (ACTC1) in iCMs were quantified and compared with those in control cells.

Cardiomyocyte-specific marker expression in the differentiated cardiomyocytes, at protein level, was analyzed by immunocytochemistry. Differentiated iCMs and undifferentiated DFCs were permeabilized with $0.2 \%$ Triton $X-100$ supplemented with $2 \%$ bovine serum albumin (BSA) for $30 \mathrm{~min}$. The cells were then fixed with $3.7 \%$ paraformaldehyde for hr and blocked in D-PBS with $2 \%$ BSA for $1 \mathrm{~h}$. Cells were incubated with primary antibodies against cardiomyocyte specific a-SMA (ab7817, mouse monoclonal; Abcam, Cambridge, UK, 1:200), TNNT2 (ab125266, rabbit polyclonal; Abcam, 1:200), DESMIN (sc14026, rabbit polyclonal; Santa Cruz, 1:200), and ACTC1 (A9357, mouse monoclonal; Sigma-Aldrich, $1: 200$ ) at $4^{\circ} \mathrm{C}$ for $12 \mathrm{~h}$. Cells were then rinsed thrice with DPBS followed by incubation with FITC-conjugated secondary antibodies (donkey anti-rabbit IgG, Jackson Immunoresearch, West Grove, PA, USA; goat anti-mouse $\operatorname{IgG} / \operatorname{IgM}, \quad B D$ Pharmingen $^{\mathrm{TM}}$ ) or Alexa fluor ${ }^{\circledR} 594$ conjugated secondary antibodies (A17045, goat anti-rabbit IgG, Jackson Immunoresearch, 1:200) for $1 \mathrm{~h}$. Finally, cell nuclei were counterstained with $1 \mu \mathrm{g} / \mathrm{ml}$ 4',6-diamidino-2-phenylindole (DAPI; Vectasheid ${ }^{\circledR}$, Vector Lab, Burlingame, CA, USA) for $5 \mathrm{~min}$ at room temperature.

\section{In vivo homing capacity of induced cardiomyocytes from DFCs}

For in vivo tracking of the homing, 
iCMs were labeled with PKH26 (PKH26GL, Sigma-Aldrich, USA) according to manufacturer's protocol and earlier reports $[6,10]$. Briefly, iCMs were trypsinized and washed twice with DPBS. The iCMs were suspended in diluent " $C$ ". Equal volumes of cell suspension and PKH26 were mixed and incubated at room temperature for $5 \mathrm{~min}$ at the end of which, serum containing media were added to the samples to stop the reaction and washed thrice with DPBS.

To check the homing capacity of the differentiated cells, $1 \times 10^{6}$ PKH26 labeled iCMs in 100 $\mu \mathrm{L}$ of PBS were injected intraperitoneally into each of six BALB/c mice (males, aged 8-12 weeks, Charles River, Orient Bio Inc., Sungnam, Korea). Two groups of three mice each served as control groups and received a $100 \mu \mathrm{L}$ of PBS injection or no injection. At 14 days after systemic cell delivery, the experimental animals were anesthetized and about $200 \mu \mathrm{L}$ of blood was drawn for serum preparation. The animals were then euthanized by $\mathrm{KCl}$ injection. Hearts, livers, and kidneys were recovered and tissue sections were prepared for the detection of PKH26 fluorescence, as described earlier [6, 10]. Briefly, tissues were embedded in optimal cutting temperature compound (Tissue-Tek, Sakura Finetechincal Co., Ltd., Tokyo, Japan), rapidly frozen and cut into sections of $4 \mu \mathrm{m}$ thickness using Cryocut (Leica CM3050S, Leica, Wetzlar, Germany). The sections from each tissue were mounted on glass slides and stained with DAPI, which stains all the cells. The stained cells and PKH26 fluorescence $(567 \mathrm{~nm})$ were observed under a fluorescence microscope (BX51, Olympus, Tokyo, Japan) equipped with a fluorescent digital camera (DP72, Olympus). Both PKH26 and DAPI positive cells were counted in each section to calculate the ratio of PKH26 positive cells to the total number of cells. Five different tissue sections of each organ were examined.

To analyze the in vivo immune response to the injected cells, serum was separated from the blood collected from the experimental animals before euthanization. The serum levels of interleukin-2 (IL-2) and interleukin-10 (IL-10) were measured using commercially available ELISA kit (KMC0101, Invitrogen). These values were compared with those of the two control groups.

\section{Statistical analysis}

Statistical analysis of the gene expression results was performed by one-way analysis of variance (ANOVA), followed by Tukey's test for multiple comparisons or an unpaired t-test for single comparisons of experimental data relative to the control values, using PASW statistics 18 (SPSS Inc., company, Country). Results are expressed as mean \pm standard deviation, and differences were considered significant at $\mathrm{p}<0.05$.

\section{Results}

\section{Characterization of DFCs}

DFCs were isolated from the dental follicles of the extracted wisdom teeth and cultured. After 7 days of initial plating, fibroblast-like colonies were observed in the plates which became homogenous at passage 3 (Figure 1A). The expression of early transcription factors, Oct4, Sox2, and Nanog, was detected in the cultured DFCs by RT-PCR (Figure 1B). In addition, DFCs were found positive for the expression of mesenchymal stem cell markers (CD44, CD90, and CD105) and negative for hematopoietic markers (CD34 and CD45) (Figure 2). PDT analysis showed steady increase in cell growth up to 12 days (Figure 1C).

To assess the differentiation potential, DFCs from passage 3 were induced to differentiate into osteocytes and adipocytes in vitro. After 21 days of induction, DFCs successfully differentiated into osteocytes and adipocytes; this was confirmed by cytochemical staining. The adipogenic differentiation was confirmed by staining with Oil red O (Figure 3A), followed by measuring the expression of adipocyte specific markers, FABP4, LPL and PPARY by RT-qPCR. Expression of these genes was approximately 4-8 fold higher in the differentiated cells (DF) compared to the non-differentiated DFCs $(\mathrm{NDF}) \quad(\mathrm{p}<0.05)$ (Figure 3B). Osteogenic differentiation was confirmed by staining with Alizarin red and von Kossa stain (Figure 3A), and by the significant increase in the mRNA expression of osteogenic specific genes, ON, RUNX-2 and BMP-2 (Figure 3B). Osteogenic DFCs showed about 10-20 times higher levels of these mRNAs compared to NDFs $(p<0.05)$ (Figure 3B).

\section{In vitro induction of DFC differentiation into cardiomyocytes by SAHA}

The morphological changes of DFCs undergoing cardiomyogenic differentiation by SAHA exposure could be observed from 7 days after induction. The cell morphology changed continuously - elongated with extended cytoplasmic processes. Spontaneous beating cells could not be found during the entire cell differentiation period of 14 days (Figure 4A). After cardiomyogenic induction of DFCs for 14 days, the iCMs were collected and analyzed for the expression of cardiomyocyte specific markers, including TNNT2, a-SMA, Desmin, and ACTC1 by RT-qPCR. The mRNA levels of these cardiogenic markers were significantly higher in DF cells compared to those in NDF cells; 
approximately 3-5 fold higher in DF (Figure 4B) (p < $0.05)$. Furthermore, the expression at protein level of these cardiomyocyte markers, TNNT2, a-SMA, DESMIN, and ACTC1, was also higher in iCMs as shown by immunocytochemical analysis (Figure 5).
These results indicate that exposure of DFCs to SAHA induces cardiomyogenic differentiation and expression of cardiac specific markers, both at mRNA and protein levels.
A
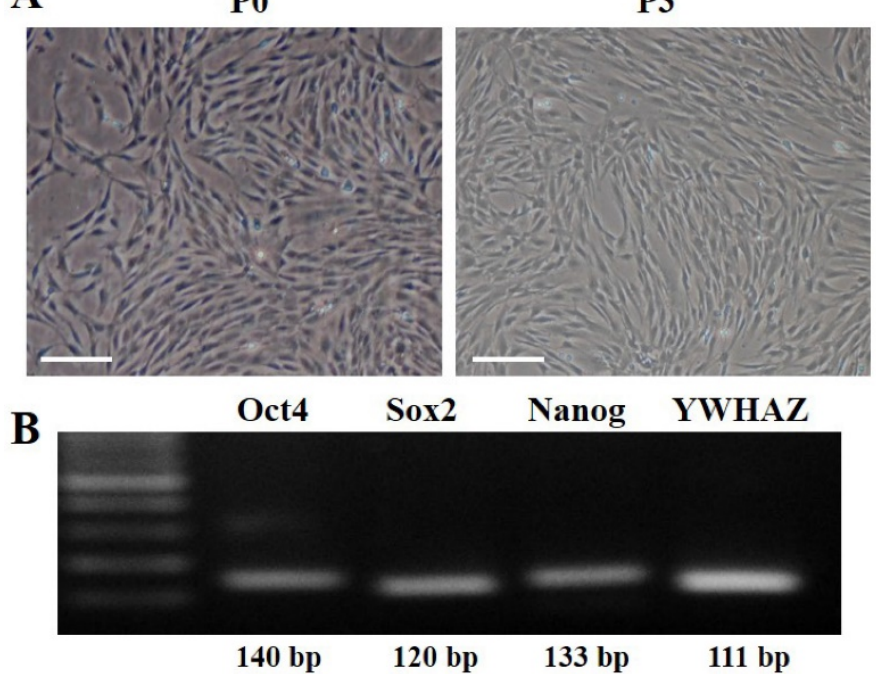

C

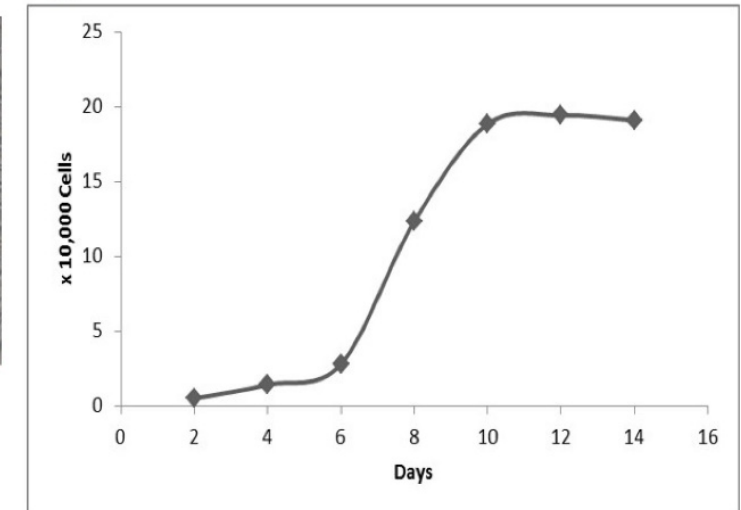

Figure 1: Culture and characterization of human dental follicle-derived stem cells (DFCs). (A) Morphology of DFCs in primary culture (P0) and at 3rd passage (P3). Plate-adhesion and fibroblast-like growth pattern were observed. Scale bar $=50 \mu \mathrm{m}$. (B) DFC at passage 3 showing expression of early transcription markers, Oct4, Sox2, and Nanog, by RT-PCR. (C) Growth curve for population doubling time (PDT) of DFCs during the 14 days of cell culture, which showed a favorable cell proliferation pattern of DFCs. Abbreviations: Oct4, octamer-binding transcription factor 4; Sox2, sex determining region Y-box 2.
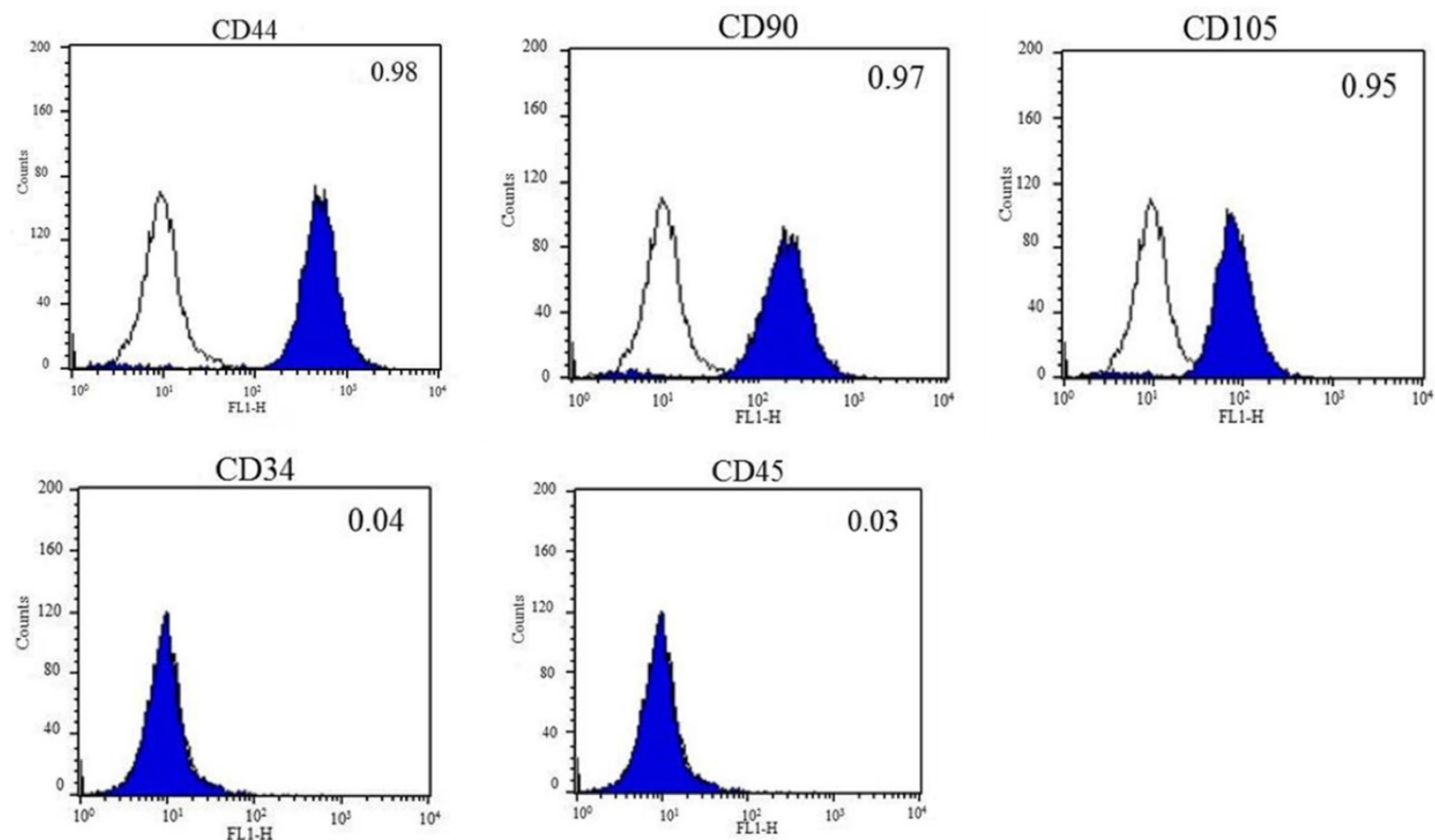

Figure 2: Fluorescent activated cell sorting (FACS) analysis of DFCs. Mesenchymal stem cell-markers, CD44, CD90, and CD105, were highly expressed, whereas the expression of hematopoietic markers, CD34 and CD45, was almost negligible in DFCs after 3 passages. The blank histograms represent an antibody isotype control and filled shaded histogram indicates specific antibodies. 
A
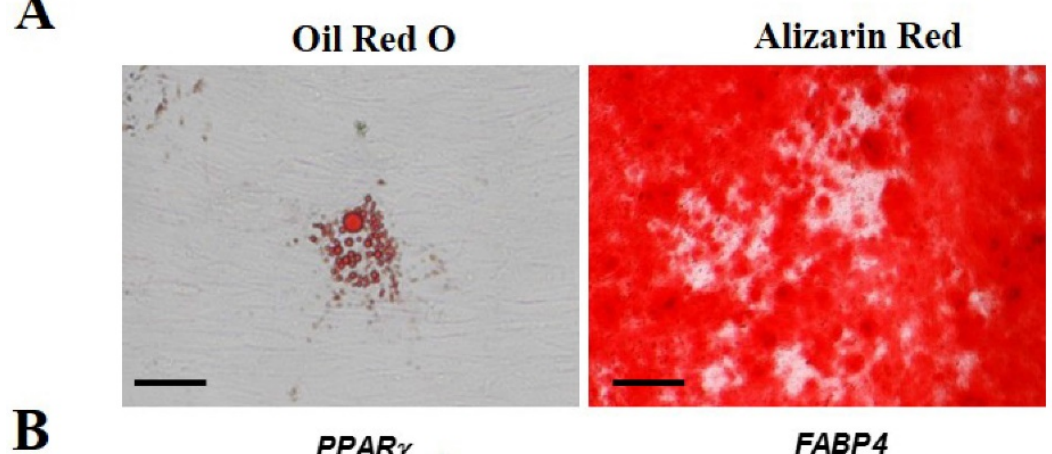

FABP4

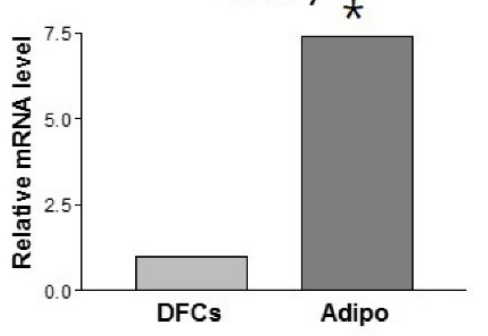

RUNX2

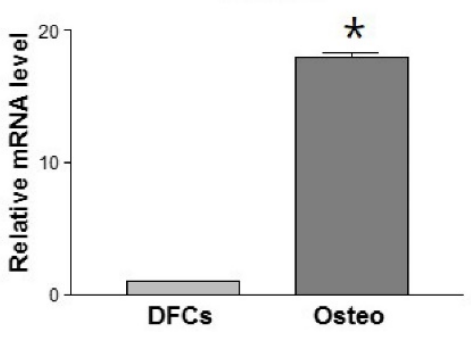

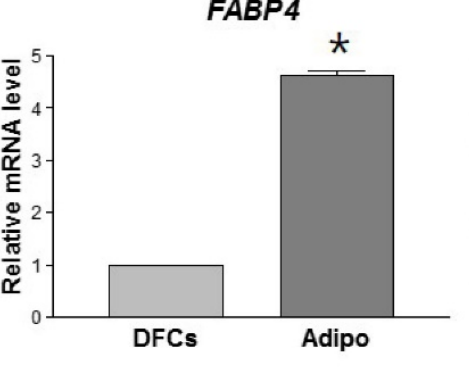

ON

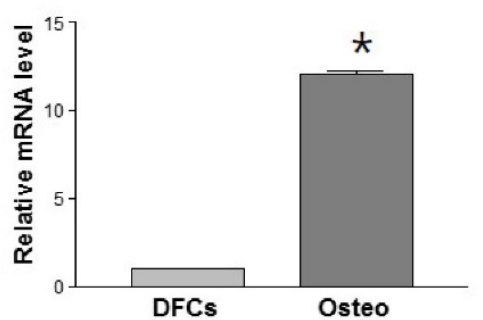

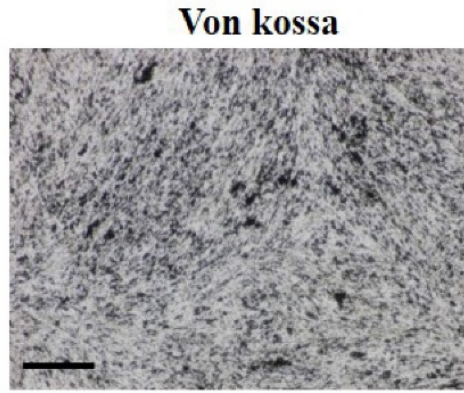

$L P L$

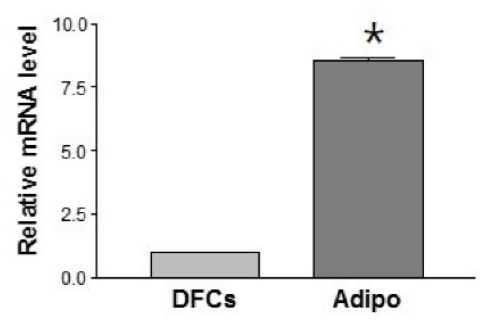

BMP2

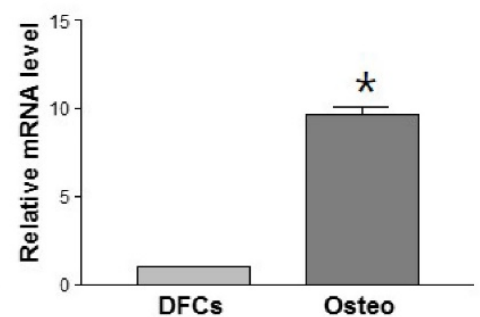

Figure 3: Lineage specific differentiation potential of DFCs. (A) Successful in vitro adipogenic and osteogenic differentiation of DFCs as identified by Oil red-O staining for lipid droplets and Alizarin red and von Kossa staining for mineralized nodules and calcium deposition, respectively. Scale bar $=100 \mu \mathrm{m}$. (B) Relative mRNA levels of differentiated cells compared to those of undifferentiated DFCs by RT-qPCR. Approximately 4-10 times and 10-20 times higher expression levels of lineage-specific mRNAs were detected in the differentiated adipogenic and osteogenic cells, respectively, compared to those in undifferentiated DFCs. Asterisk $(*)$ represents statistically significant differences between the two groups $(\mathrm{p}<0.05)$. Abbreviations: PPAR $\gamma$, peroxisome proliferative activated receptor gamma; FABP4, fatty-acid-binding protein 4; LPL, lipoprotein lipase; RUNX2, runt related transcription factor 2; ON, osteonectin; BMP2, bone morphogenetic protein 2.

\section{In vivo homing and immune response after systemic injection of $\mathrm{iCMs}$}

The experimental mice were euthanized after 14 days of intraperitoneal injection of PKH26 labeled iCMs, and different organs (heart, liver, and kidney) were examined for the presence of the injected cells. Fluorescence microscopy detected PKH26 labeled cells in heart, liver, and kidney sections (Figure 6). The ratios of PKH26 positive cells to the total number of injected cells were $5.6 \pm 1.0 \%, 3.6 \pm 1.1 \%$, and $11.6 \pm$ $2.7 \%$ in heart, liver, and kidney, respectively. These results indicate that the intraperitoneally injected iCMs homed to and colonized these organs, survived and proliferated for 14 days after the injection, demonstrating the homing potential of the iCMs into various organs including the heart muscle.

Serum levels of IL-2 and IL-10 were analyzed at 14 days after the transplantation, to assess the immune and inflammatory responses to the injected cells. There was no significant difference in serum levels of IL-2 and IL-10 between the iCM injection group and the two control groups $(p>0.05)$ (Figure 7 ), indicating that there was no major inflammatory or immune response in vivo to the systemic transplantation of differentiated cardiomyocytes.

\section{Discussion}

Human DFCs were isolated from the dental follicles of extracted wisdom teeth. These cells exhibited fibroblast-like appearance and plate adherent growth pattern. DFCs were positive for the expression MSC markers (CD44, CD90, and CD105) by FACS analysis and stemness markers (Nanog, Oct4, and Sox2) by RT-qPCR. The DFCs successfully differentiated into osteocytes and adipocytes with high cell proliferation rate, under appropriate culture conditions. These results indicate that the DFCs isolated in the present study possess MSC characteristics, as those in our previous studies [6, 8-10]. The DFCs differentiated successfully in vitro 
into cardiomyocytes in media containing SAHA. The differentiated cells were morphologically similar to in vivo cardiomyocytes and expressed abundantly cardiomyocyte-specific markers, both at the mRNA and protein level. Similar to this study, an earlier study reported that the dental stem cells from deciduous pulp could differentiate into cardiomyocytes in vitro under activin A and BMP2 induction protocol [11].

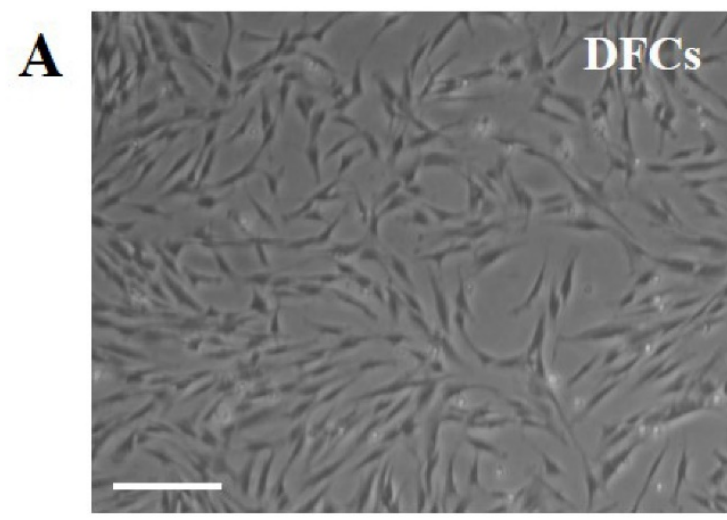

ACTC1

B

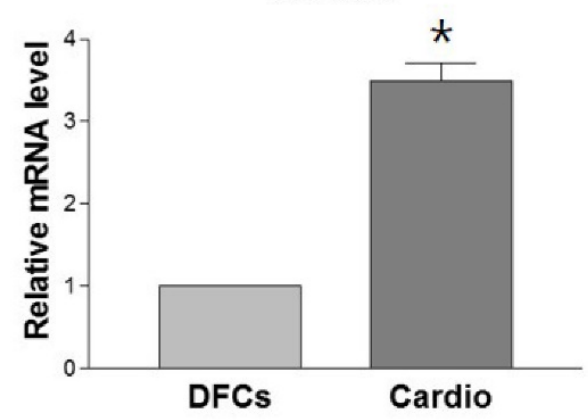

Desmin

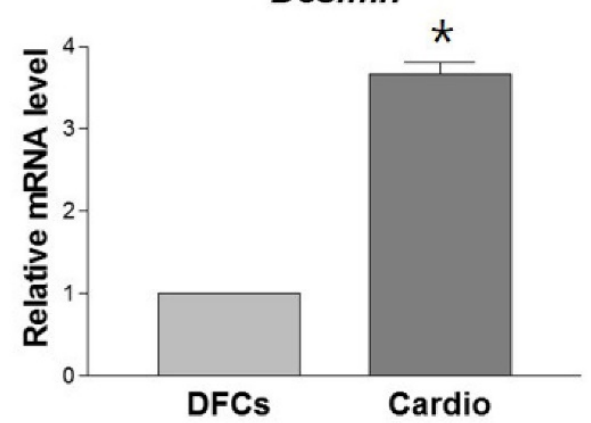

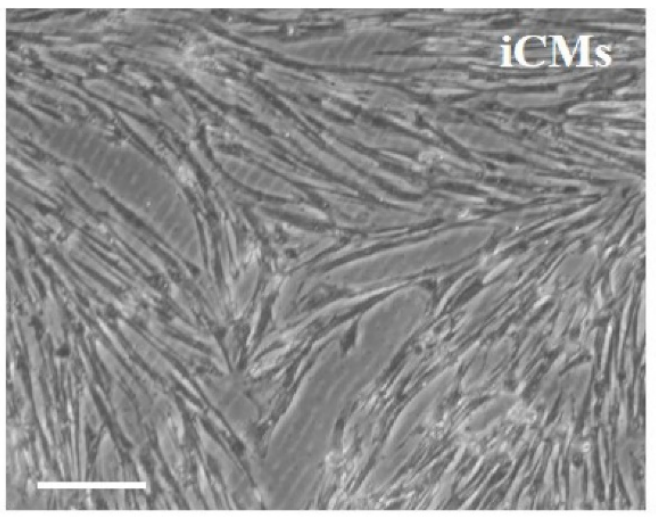

$\alpha$-SMA

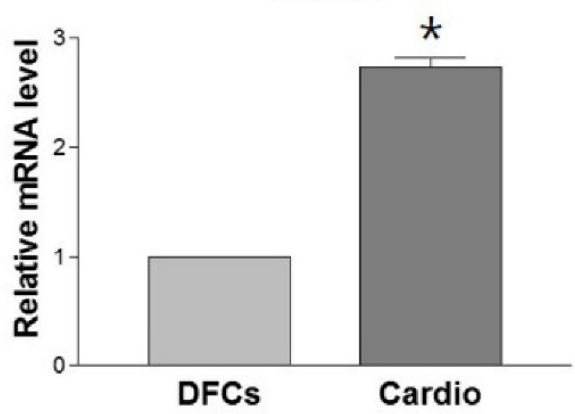

TNNT2

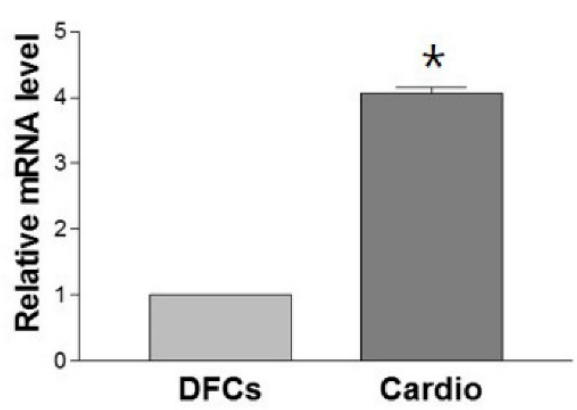

C Ladder TNNT2 Desmin $\quad A C T C 1 \quad \boldsymbol{\alpha}-S M A$

Figure 4: In vitro differentiation of DFCs into cardiomyocytes in SAHA containing media. (A) After 14 days of SAHA induction, the induced cardiomyocytes (iCMs) showed more elongated cell morphology with extended cytoplasmic processes compared to the undifferentiated DFCs. Scale bar $=50 \mu \mathrm{m}$. (B) Relative mRNA levels of cardiomyocyte-specific markers, TNNT2, $\alpha$-SMA, Desmin, and ACTCI, were approximately 3-5 times higher in the iCMs compared to those in DFCs. Data represent the mean \pm standard deviation of five independent experiments. *significantly different from control (DFCs) ( $<<0.05)$. (C) Representative images of RT-qPCR products to show product sizes from (B). Abbreviations: $\alpha$-SMA, alpha-smooth muscle actin; TNNT2, Cardiac muscle troponin T; ACTC1, Cardiac muscle alpha actin. 


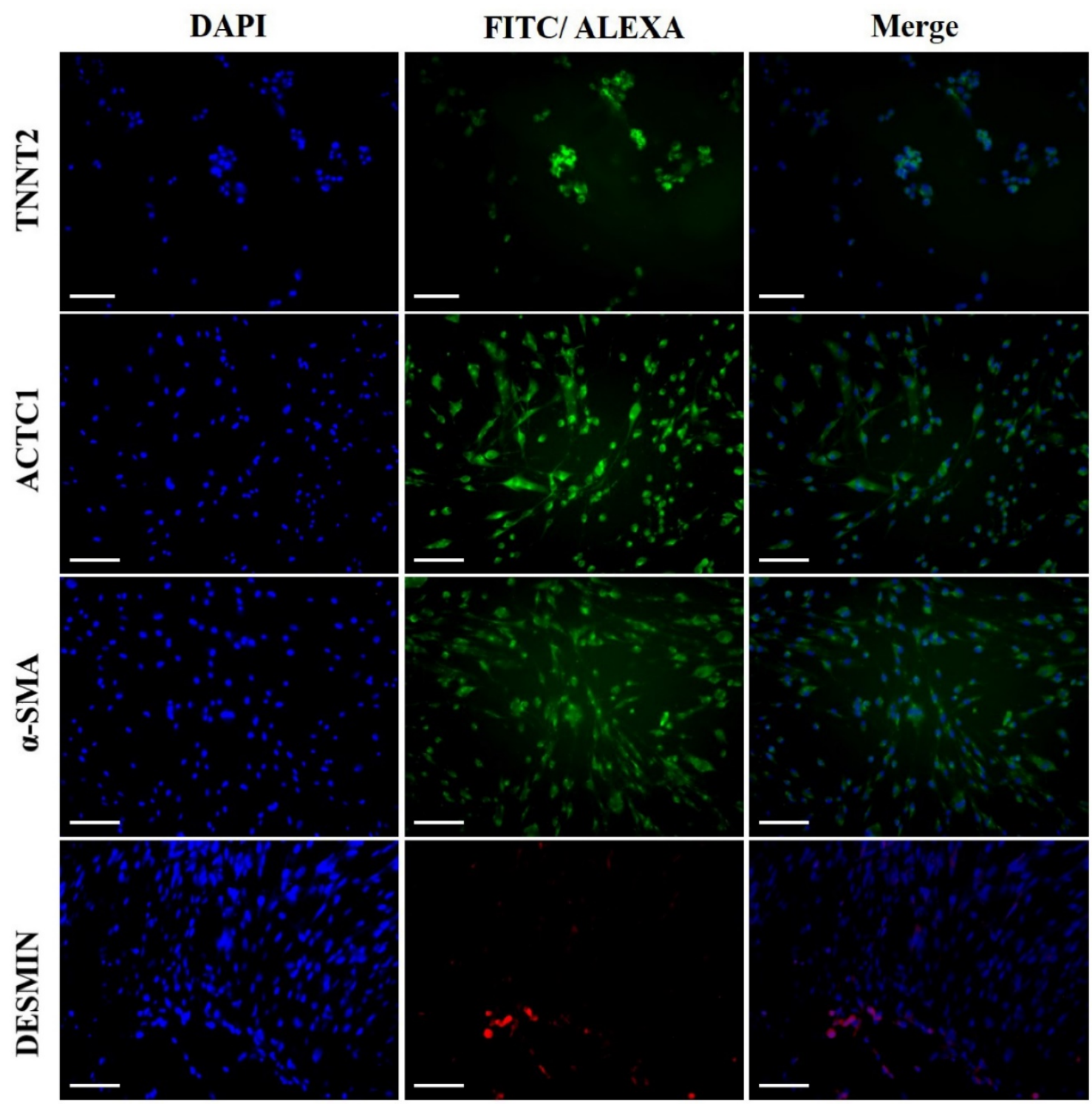

Figure 5: Immunocytochemical analysis of the iCMs for cardiomyocyte specific markers. Abundant expressions of TNNT2, $\alpha$-SMA, DESMIN, and ACTC1 were detected in the iCMs after 7 days of induction by SAHA. Scale bar $=100 \mu \mathrm{m}$.

To our knowledge, this is the first time that the stem cells from dental tissue were differentiated into cardiomyocytes using SAHA induction protocol. Most studies on in vitro cardiomyogenic differentiation of stem cells have used bone marrow-derived MSCs as the cell source and 5-aza as the induction chemical $[4,12-14]$. 5-aza is a DNA methylation inhibitor and one of the most commonly used chemicals to induce the differentiation of stem cells into myocardial cells, probably by the activation of some of the dormant genes through DNA demethylation, tough the exact mechanism has not yet been elucidated $[4,12,17]$. However, the rate of 5 -aza induced myocardial differentiation was extremely low and proved cytotoxic to the differentiating cells resulting in cell death [14-16]. SAHA, a member of histone deacetylase (HDAC) inhibitor family, was another chemical with the potential to induce the differentiation of stem cells into cardiomyocytes [16]. HDAC inhibitors have been studied as novel therapies for inflammation, cancer, neurodegeneration, and heart failure [23]. The balance of acetylation and deacetylation of nuclear histones is crucial for the regulation of gene expression and maintenance of the chromatin structure and function [24]. Inhibition of HDAC by SAHA results in hyper-acetylation of histone, leads to cell growth arrest and apoptosis in various tumor cells [25, 26]. SAHA has been known to inhibit all the 11 known class I and class II HDACs [16]. Class II HDACs have been shown to play an important role in cardiac development and cardiac hypertrophy, and could regulate the entry of mesoderm cells into cardiomyoblastogenesis [27, 28]. 


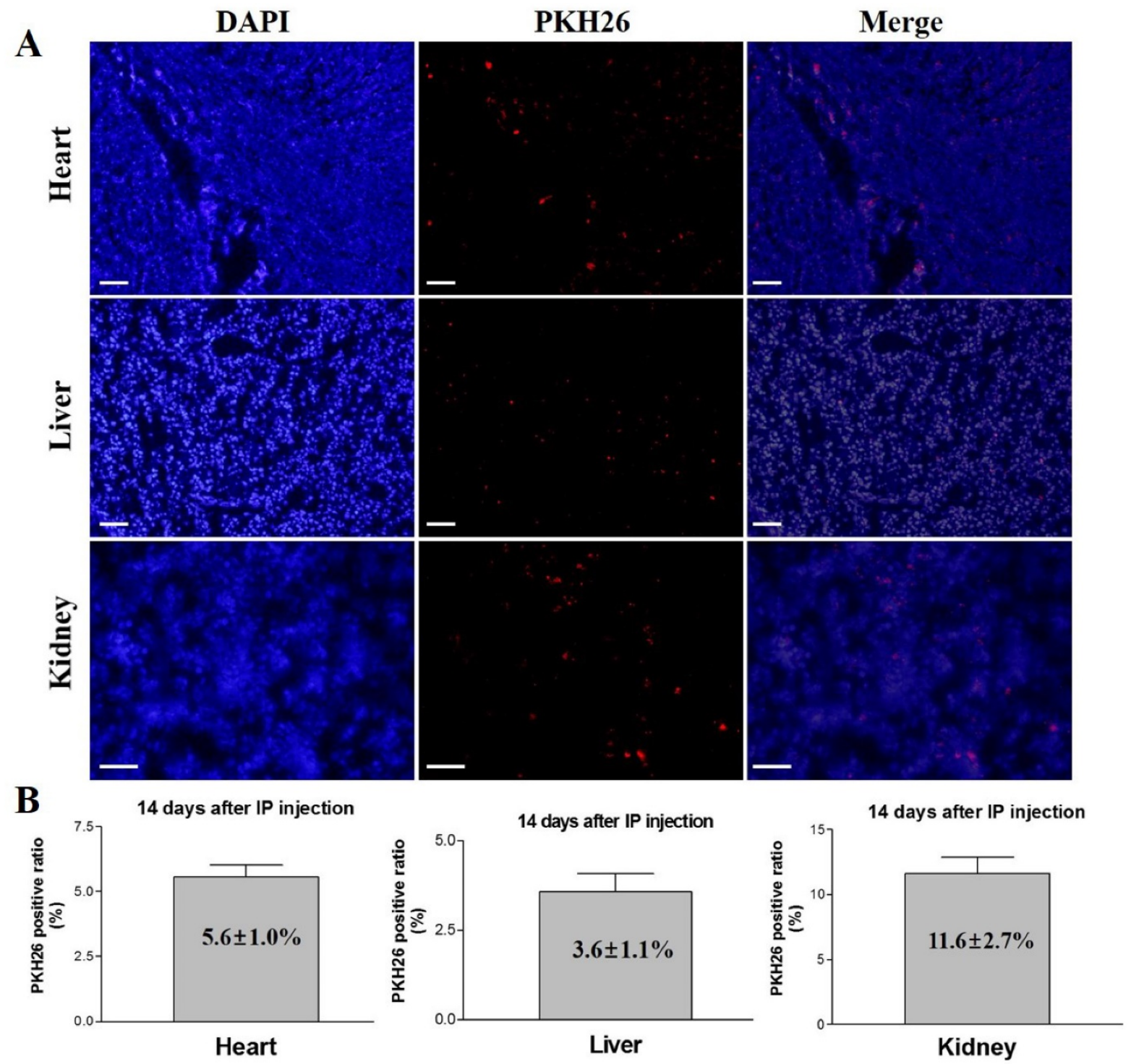

Figure 6: In vivo tracking and homing analysis after systemic administration of PKH26 labeled iCMs. At 14 days after IP injection of the cells, PKH26 positive cells were detected in the heart, liver, and kidney tissues. The PKH26 positive ratios were $5.6 \pm 1.0 \%$ in heart, $3.6 \pm 1.1 \%$ in liver, and $11.6 \pm 2.7 \%$ in kidney. Scale bar $=100 \mu \mathrm{m}$.

A

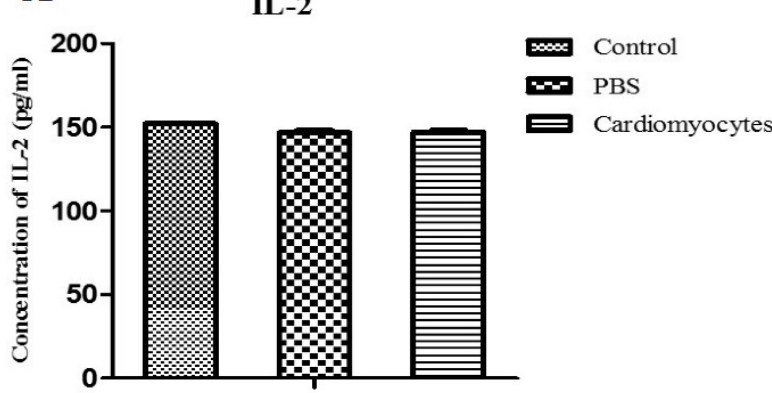

B

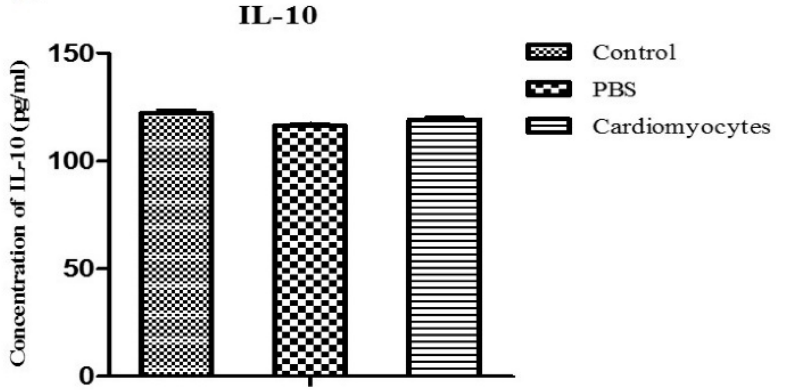

Figure 7: Comparative analysis of the serum levels of IL-2 and IL-10 in the systemic iCM injected mice and controls. The serum levels of IL-2 and IL-10 in the experimental mice ( 14 days after IP injection of $\mathrm{iCM}$ ) were not different compared to those of the two control groups.

In the present study, the cells differentiated from DFCs by treating with SAHA for 14 days, exhibited morphological changes such as elongated stick like appearance with extended cytoplasmic processes. Similar morphological changes were reported in other studies describing the cardiomyogenic differentiation of stem cells by chemical induction [11-17, 29, 30]. The cells differentiated by SAHA induction showed abundant expression of cardiomyocyte-specific markers, such as a-SMA, TNNT2, Desmin, and 
ACTC1, at both mRNA and protein levels. Although the beating cells could not be found in the present study, these results demonstrate that the SAHA induction protocol can successfully differentiate DFCs into cardiomyocytes in vitro. This result is in agreement with those of the previous studies, which showed successful in vitro cardiomyogenic differentiation of human fetal liver and bone marrow derived mesenchymal stem cells, but no beating cells $[16,17,30]$.

The homing properties of the systemically administered therapeutic cells have been studied to improve the efficiency of cell therapy in regenerative medicine. Following systemic administration, the injected cells migrate to target organ or tissue, and produce paracrine and juxtacrine effects, which enhance the regenerative efficiency of endogenous cells [31]. In a recent report, intraperitoneally injected dental pulp stem cells showed homing capacity into kidneys $(33.25 \%)$, liver $(9.10 \%)$, and lungs $(1.85 \%)$ at 3 days after injection [21]. In another study, intravenously injected bone marrow stem cells showed lower homing capacity; only $3.42 \%$ in lung and $0.50 \%$ in spleen at 14 days after cell injection [20]. Injected MSCs showed 2.0-22.0\% of cell engraftment into kidneys $[32,33]$. These differences in the rates of stem cell homing could be correlated to the differences in experimental conditions such as the injected cell type (BMSC or other sources), injection method (IV, IP, or intra-arterial), condition of recipient animals (healthy or disease model), survival period after cell injection, detection method (Immunohistochemistry or Flow cytometry), etc. In contrast to the other studies, PKH26 labeled iCMs were systemically injected into healthy mice in the present study. Notably, 14 days after the IP injection, labeled iCMs were detected in the tissues of the kidney, liver, and heart, at ratios of approximately $11.6 \%, 3.6 \%$, and $5.6 \%$, respectively. These homing ratios were relatively low in the kidney and liver compared to the results reported in an earlier study [21], which could be correlated with the longer animal survival time (14 days) after cell injection in this study. In addition, the cells injected in our study were differentiated iCMs and not the undifferentiated MSCs, which might have limited or completely precluded active proliferation of cells entrapped in the kidney and liver. However, we presume that in spite of the small number of injected iCMs that homed to heart muscle, they could proliferate for 14 days and reach the observed ratio of 5-6\%. In addition, the low homing efficiency into the heart tissue might be related to the experimental animal model used in the present study, healthy mice rather than the heart disease model. Despite this limitation, the results from this study suggest that the systemic administration of the iCMs from DFCs can be used as an effective cell therapy method for myocardial heart diseases. To the best of our knowledge, the present study is the first to demonstrate the homing of cardiomyocytes differentiated from MSCs following systemic cell administration.

We did not observe any significant difference in serum levels of IL-2 and IL-10 between the iCM injection group and the two control groups. This result demonstrates that the transplanted iCMs did not elicit any inflammatory response in vivo. We have showed in an earlier report, the immunomodulatory properties of DFCs such as the extremely low expression of major histocompatibility complex class II and decreased expressions of CD3 and CD4 when transplanted into tissues in a localized manner [10]. Interestingly, although the cell type (iCMs in this study vs. DFCs) and cell administration method (systemic injection in this study vs. localized transplantation) were different from the earlier study, the anti-inflammatory and immunomodulatory effects observed in both the studies were similar.

In conclusion, human dental follicle-derived stem cells (DFCs), which exhibit MSC characteristics, differentiated successfully into cardiomyocytes in vitro under the influence of SAHA in the culture media. These iCMs homed to heart muscle following systemic cell injection in experimental animals. The injected cells did not elicit any inflammatory response. These results demonstrate that DFCs can be an excellent source for cardiomyocyte differentiation and regeneration. Moreover, the iCMs from DFCs can be delivered into heart muscle via systemic administration, including intravenous and/or intraperitoneal injection. Further studies are needed to confirm these results, including iCM injection in heart disease models and investigating the possibility of additional side effects following systemic cell administration.

\section{Abbreviations}

Oct4, octamer-binding transcription factor 4; SOX2, sex determining region Y-box 2; FABP4, fatty-acid-binding protein 4; LPL, lipoprotein lipase, PPARY, peroxisome proliferative activated receptor gamma; BMP2, bone morphogenetic protein 2; ON, osteonectin; RUNX2, runt related transcription factor 2; TNNT2, Cardiac muscle troponin T; a-SMA, alpha-smooth muscle actin; ACTC1, Cardiac muscle alpha actin; YWHAZ, Tyrosine 3-monooxygenase/ tryptophan 5-monooxygenase activation protein, zeta. 


\section{Acknowledgement}

This work was supported by the National Research Foundation of Korea (NRF) Grant funded by the Korean Government (NRF-2014R1A1A2058807).

\section{Author's contributions}

Iel-Yong Sung and Han-Na Son participated in designing the present study, performed data analysis, and contributed to the manuscript preparation. Imran Ullah, Dinesh Bharti, and Ju-Mi Park performed cell culture and in vitro cardiomyogenic differentiation experiments. Young-Cheol Cho, June-Ho Byun, Young-Hoon Kang, and Su-Jin Sung collected the extracted wisdom teeth, participated in in vivo animal experiments, and performed the statistical analysis. Jong-Woo Kim analyzed the in vitro differentiated cardiomyocytes and in vivo homing specimens. Gyu-Jin Rho and Bong-Wook Park made equal contributions to the management of all the experiments, analysis of the collected data, and were major contributors to the manuscript preparation. All the authors read and approved the final manuscript.

\section{Competing Interests}

The authors have declared that no competing interest exists.

\section{References}

1. Schueller PO, Meyer C, Brehm M, Wernet P, Schannwell CM, Strauer BE. Intracoronary autologous bone marrow cell transplantation beneficially modulates heart rate variability. Int J Cardiol. 2007;119(3):398-9

2. Fedak PW, Verma S, Weisel RD, Skrtic M, Li RK. Cardiac remodeling and failure: from molecules to man (Part III). Cardiovasc Pathol. 2005;14(3):109-19.

3. Psaltis PJ, Zannettino AC, Worthley SG, Gronthos S. Concise review: mesenchymal stromal cells: potential for cardiovascular repair. Stem Cells. 2008;26(9):2201-10.

4. Shen H, Wang Y, Zhang Z, Yang J, Hu S, Shen Z. Mesenchymal Stem Cells for Cardiac Regenerative Therapy: Optimization of Cell Differentiation Strategy. Stem Cells Int. 2015;2015:524756.

5. Kang EJ, Byun JH, Choi YJ, Maeng GH, Lee SL, Kang DH, et al. In vitro and in vivo osteogenesis of porcine skin-derived mesenchymal stem cell-like cells with a demineralized bone and fibrin glue scaffold. Tissue Eng Part A. 2010;16(3):815-27.

6. Park BW, Kang EJ, Byun JH, Son MG, Kim HJ, Hah YS, et al. In vitro and in vivo osteogenesis of human mesenchymal stem cells derived from skin, bone marrow and dental follicle tissues. Differentiation. 2012;83(5):249-59.

7. Ge D, Liu X, Li L, Wu J, Tu Q, Shi Y, et al. Chemical and physical stimuli induce cardiomyocyte differentiation from stem cells. Biochem Biophys Res Commun. 2009;381(3):317-21.

8. Jeon BG, Kang EJ, Kumar BM, Maeng GH, Ock SA, Kwack DO, et al Comparative analysis of telomere length, telomerase and reverse transcriptase activity in human dental stem cells. Cell Transplant. 2011;20(11-12):1693-705.

9. Park BW, Jang SJ, Byun JH, Kang YH, Choi MJ, Park WU, et al. Cryopreservation of human dental follicle tissue for use as a resource of autologous mesenchymal stem cells. J Tissue Eng Regen Med. 2014.

10. Kang YH, Lee HJ, Jang SJ, Byun JH, Lee JS, Lee HC, et al. Immunomodulatory properties and in vivo osteogenesis of human dental stem cells from fresh and cryopreserved dental follicles. Differentiation. 2015;90(1-3):48-58.

11. Loo ZX, Kunasekaran W, Govindasamy V, Musa S, Abu Kasim NH. Comparative analysis of cardiovascular development related genes in stem cells isolated from deciduous pulp and adipose tissue. ScientificWorldJournal. 2014;2014:186508.

12. Wakitani S, Saito T, Caplan AI. Myogenic cells derived from rat bone marrow mesenchymal stem cells exposed to 5-azacytidine. Muscle Nerve. 1995;18(12):1417-26.

13. Makino S, Fukuda K, Miyoshi S, Konishi F, Kodama H, Pan J, et al. Cardiomyocytes can be generated from marrow stromal cells in vitro. J Clin Invest. 1999;103(5):697-705.
14. Gao Q, Guo M, Jiang X, Hu X, Wang Y, Fan Y. A cocktail method for promoting cardiomyocyte differentiation from bone marrow-derived mesenchymal stem cells. Stem Cells Int. 2014;2014:162024.

15. Fukuda K, Yuasa S. Stem cells as a source of regenerative cardiomyocytes. Circ Res. 2006;98(8):1002-13.

16. Feng C, Zhu J, Zhao L, Lu T, Zhang W, Liu Z, et al. Suberoylanilide hydroxamic acid promotes cardiomyocyte differentiation of rat mesenchymal stem cells. Exp Cell Res. 2009;315(17):3044-51.

17. Deng F, Lei H, Hu Y, He L, Fu H, Feng R, et al. Combination of retinoic acid, dimethyl sulfoxide and 5-azacytidine promotes cardiac differentiation of human fetal liver-derived mesenchymal stem cells. Cell Tissue Bank. 2016;17(1):147-59.

18. Chen Y, Du J, Zhao YT, Zhang L, Lv G, Zhuang S, et al. Histone deacetylase (HDAC) inhibition improves myocardial function and prevents cardiac remodeling in diabetic mice. Cardiovasc Diabetol. 2015;14:99.

19. Schaun MI, Eibel B, Kristocheck M, Sausen G, Machado L, Koche A, et al. Cell Therapy in Ischemic Heart Disease: Interventions That Modulate Cardiac Regeneration. Stem Cells Int. 2016;2016:2171035.

20. Jiang L, Song XH, Liu P, Zeng CL, Huang ZS, Zhu LJ, et al. Platelet-mediated mesenchymal stem cells homing to the lung reduces monocrotaline-induced rat pulmonary hypertension. Cell Transplant. 2012;21(7):1463-75.

21. Barros MA, Martins JF, Maria DA, Wenceslau CV, De Souza DM, Kerkis A, et al. Immature Dental Pulp Stem Cells Showed Renotropic and Pericyte-Like Properties in Acute Renal Failure in Rats. Cell Med. 2015;7(3):95-108.

22. Figueroa FE, Carrion F, Villanueva S, Khoury M. Mesenchymal stem cell treatment for autoimmune diseases: a critical review. Biol Res. 2012;45(3):269-77.

23. $\mathrm{Xu} \mathrm{Q}$, Lin $\mathrm{X}$, Andrews L, Patel D, Lampe PD, Veenstra RD. Histone deacetylase inhibition reduces cardiac connexin 43 expression and gap junction communication. Front Pharmacol. 2013;4:44.

24. Mei S, Ho AD, Mahlknecht U. Role of histone deacetylase inhibitors in the treatment of cancer (Review). Int J Oncol. 2004;25(6):1509-19.

25. Kouraklis G, Theocharis S. Histone deacetylase inhibitors and anticancer therapy. Curr Med Chem Anticancer Agents. 2002;2(4):477-84

26. Santini V, Gozzini A, Ferrari G. Histone deacetylase inhibitors: molecular and biological activity as a premise to clinical application. Curr Drug Metab. 2007;8(4):383-93.

27. McKinsey TA, Olson EN. Dual roles of histone deacetylases in the control of cardiac growth. Novartis Found Symp. 2004;259:132-41; discussion 41-5, 63-9.

28. Karamboulas C, Swedani A, Ward C, Al-Madhoun AS, Wilton S, Boisvenue S, et al. HDAC activity regulates entry of mesoderm cells into the cardiac muscle lineage. J Cell Sci. 2006;119(Pt 20):4305-14.

29. Antonitsis P, Ioannidou-Papagiannaki E, Kaidoglou A, Charokopos N, Kalogeridis A, Kouzi-Koliakou K, et al. Cardiomyogenic potential of human adult bone marrow mesenchymal stem cells in vitro. Thorac Cardiovasc Surg. 2008;56(2):77-82.

30. Siegel G, Krause P, Wohrle S, Nowak P, Ayturan M, Kluba T, et al. Bone marrow-derived human mesenchymal stem cells express cardiomyogenic proteins but do not exhibit functional cardiomyogenic differentiation potential. Stem Cells Dev. 2012;21(13):2457-70.

31. Sohni A, Verfaillie CM. Mesenchymal stem cells migration homing and tracking. Stem Cells Int. 2013;2013:130763.

32. Herrera MB, Bussolati B, Bruno S, Morando L, Mauriello-Romanazzi G, Sanavio F, et al. Exogenous mesenchymal stem cells localize to the kidney by means of CD44 following acute tubular injury. Kidney Int. 2007;72(4):430-41.

33. Perin L, Sedrakyan S, Giuliani S, Da Sacco S, Carraro G, Shiri L, et al. Protective effect of human amniotic fluid stem cells in an immunodeficient mouse model of acute tubular necrosis. PLoS One. 2010;5(2):e9357. 